\title{
La política del Ministerio de Salud de Chile para el fortalecimiento del patrimonio cultural de la salud
}

PATRICIO HEVIA ${ }^{(1)}$

El Ministerio de Salud ha asumido como una Política Nacional permanente la responsabilidad, a través de la Unidad de Patrimonio Cultural de la Salud, de identificar, proteger, conservar, poner en valor y transmitir a las futuras generaciones el valioso Patrimonio Cultural del Sistema Público de Salud de Chile

A partir del año $2000 \mathrm{y}$ en forma progresiva se ha ido avanzando en las diversas estructuras del Ministerio de Salud y de su Sistema Nacional de Servicios de Salud para el reconocimiento y valorización progresiva del riquísimo patrimonio cultural tangible e intangible de la salud chilena. Todo ello en el contexto de nuestra América Latina y el Caribe.

Para responder a los desafíos al Bicentenario se ha propuesto fortalecer la Red Chilena de Historia y Patrimonio Cultural de la Salud y poner en operación el Centro Nacional de Recuperación y Difusión del Patrimonio Cultural de la Salud.

\section{ANTECEDENTES}

El desarrollo de la Salud Pública requiere considerar sus antecedentes históricos junto con la revalorización de su Patrimonio Cultural construido a través de varias generaciones. Esto es coherente con la urgente necesidad de fortalecer la cultura en las instancias de servicio público.

El trabajo relacionado con el rescate y la recuperación de nuestro patrimonio histórico y cultural de la salud no ha sido fácil. Hay muchas prioridades en la contingencia y escasos momentos abiertos a la reflexión de un pasado que tiene significado en el presente y perspectivas de proyección hacia el futuro.

Las actividades preliminares en la recuperación progresiva del área patrimonial comenzaron en septiembre del 2000, con la creación y funcionamiento de una Comisión de Trabajo sobre el Patrimonio Cultural en Salud que funcionó ininterrumpidamente hasta el año 2007, constituida inicialmente a nivel del Gabinete de la Ministra de Salud con el objetivo de: "Reunir antecedentes de carácter histórico sobre el patrimonio cultural que posee el Sistema Nacional de Servicios de Salud, en cuanto a sus establecimientos hospitalarios, obras pictóricas, documentos y archivos, todo lo cual constituye una muestra de la atención asistencial en el ámbito de la salud pública desde principios del siglo pasado".

Considerando la importancia del rescate patrimonial en salud, en septiembre de 2002, el Ministro de Salud, mediante Resolución Exenta $\mathrm{N}^{\mathrm{o}}$ 801, creo la Unidad de Patrimonio Cultural de la Salud, dependiente de la Subsecretaría de Salud, como "una Unidad especializada para la selección e interpretación de los antecedentes históricos, así como para la administración del patrimonio cultural de la salud con una visión de conjunto". En agosto de 2003 se inauguró oficialmente, ubicándose su sede central en el Antiguo Hospital San José de Santiago, Monumento Nacional en la categoría de Monumento Histórico ${ }^{1}$.

La primera propuesta oficial sobre "Políticas y Líneas de Acción del Ministerio de Salud en Patrimonio Cultural de la Salud " fue dada a conocer en agosto de 2004 con ocasión del

(1) Unidad de Patrimonio Cultural. Ministerio de Salud. Escuela de Salud Pública. Universidad de Chile. Independencia 939. Santiago. Chile.phevia@med.uchile.cl 
Primer Encuentro Nacional de Responsables del Patrimonio Cultural de la Salud. En dicha oportunidad el lema central fue:"Construyendo juntos nuestro patrimonio en salud mirando al Bicentenario".

A partir del encuentro con participación de encargados del patrimonio cultural de los servicios de salud, Secretarías Regionales Ministeriales y Organismos Autónomos del Minsal, se creó la Red Nacional de Responsables del Patrimonio Cultural de la Salud y se firmó el "Acta de Compromiso Patrimonial en Salud" por todos los participantes y por el Ministro de Salud. Este evento y su acta patrimonial ha pasado a ser la "carta de navegación" para el desarrollo en los lugares de trabajo de las diferentes áreas temáticas del patrimonio cultural en salud, así como contribuir activamente al rescate de la memoria histórica de la salud chilena con aportes locales $\mathrm{y} / \mathrm{o}$ regionales ${ }^{2}$.

El año 2006, a raíz de las nuevas estructuras derivadas de la Reforma de la Salud, la Unidad de Patrimonio Cultural de la Salud pasó a depender de la División de Planificación Sanitaria de la Subsecretaría de Salud Pública.

\section{EL MANDATO INSTITUCIONAL}

El Ministerio de Salud le ha otorgado el mandato a la Unidad de Patrimonio Cultural de la Salud para asumir la responsable legal de profundizar el área del patrimonio histórico y cultural, tanto tangible como intangible, actuando con un enfoque intersectorial, descentralizado y participativo.

Los objetivos institucionales son los siguientes:

- Coordinar desde la Subsecretaría de Salud Pública, la investigación, el cuidado, la conservación y la administración del patrimonio cultural de la salud.

- Coordinar desde la Subsecretaría de Salud Pública, la investigación, el cuidado, la conservación y la administración del patrimonio cultural de la salud.

- Proponer a las autoridades del Ministerio de Salud los lineamientos generales para la elaboración de una política pública sobre el patrimonio cultural de la salud y las normas correspondientes para la organización, administración y gestión de dicho patrimonio.

La triple estrategia de acción patrimonial desarrollada por el Ministerio de Salud a través de su Unidad de Patrimonio Cultural se ha orientado a:

- Creación y desarrollo de la Red Nacional de Responsables del Patrimonio Cultural de Salud coordinada por la Unidad de Patrimonio Cultural de la Salud. A partir de esta experiencia se está formando actualmente la Red Chilena de Historia y Patrimonio Cultural de la Salud, integrante de la Red Latinoamericana de la BVS de Historia y Patrimonio Cultural de la Salud. Uno de los logros más significativos ha sido la institucionalización por la Ministra de Salud en mayo de 2007 del "Día del Patrimonio Cultural del Sistema Nacional de Servicios de Salud", que se celebra en todo el territorio nacional el último domingo de cada año a partir de 2008.

- Fortalecimiento de alianzas estratégicas a nivel intersectorial en patrimonio a través de diversas coordinaciones operativas con:

- Departamento de Patrimonio Arquitectónico del Ministerio de Obras Públicas (MOP).

- Consejo de Monumentos Nacionales del Ministerio de Educación.

- Dirección Nacional de Bibliotecas, Archivos y Museos del Ministerio de Educación.

- Museo Nacional de la Medicina Dr. Enrique Laval de la Facultad de Medicina de la Universidad de Chile.

- Diferentes instancias del Consejo Nacional de las Artes y la Cultura y de sus órganos regionales.

- Red de Archivos y Documentos Patrimoniales de Valparaíso, ARPA, coordinada por la Biblioteca de la Pontificia Universidad Católica de Valparaíso.

- Fundaciones y corporaciones privadas en el área patrimonial. 
- Desarrollo de áreas técnicas en la Unidad de Patrimonio Cultural de la Salud en relación a: Documentación y archivo; patrimonio arquitectónico; colecciones de objetos de valor patrimonial: investigación histórica; gestión patrimonial en salud; desarrollo de proyectos; y comunicación y difusión. Para ello, ha contado con la asesoría internacional de la Casa de Oswaldo Cruz/Fiocruz, Ministerio de Salud de Brasil; y del Centro Latinoamericano de Información en Ciencias de la Salud (BIREME/OPS-OMS) ${ }^{1}$.

Hasta el momento el Ministerio de Salud ha firmado dos convenios de colaboración en esta área temática: uno con el Consejo de Monumentos Nacionales (CMN); y otro, con la Dirección Nacional de Bibliotecas, Archivos y Museos (DIBAM), ambas instancias del Ministerio de Educación. Otro hecho destacable ha sido el Premio Conservación de Monumentos Nacionales 2006 recibido por el Ministerio de Salud y su Unidad de Patrimonio Cultural y que fuera otorgado por un equipo intersectorial dirigido por el Consejo de Monumentos Nacionales ${ }^{3}$.

\section{DESAFÍOS HACIA EL BICENTENARIO, CHILE 2010}

Para responder a los desafíos actuales y futuros de la política sanitaria chilena, se requiere rescatar y poner en valor la riqueza patrimonial histórica y cultural del Sistema Nacional de Servicios de Salud, con una mirada hacia el Bicentenario. Una de las propuestas presentadas a la Comisión Presidencial del Bicentenario Chile 2010 corresponde al proceso de fortalecimiento de la Biblioteca Virtual en Salud de Chile junto con el desarrollo de las redes sociales en historia y patrimonio de la salud, que permitan interconectar todo el territorio nacional para poner a disposición de los trabajadores de la salud y de la ciudadanía, la mejor y más completa información en salud con acceso libre y gratuito ${ }^{4-7}$.

Con este fin, se logró a inicios de 2008 la incorporación de actores institucionales de la
Salud Pública, la Historia y el Patrimonio Cultural en el Comité Consultivo Nacional de la Biblioteca Virtual en Salud (BVS) de Chile. Ha sido importante el trabajo mancomunado del Ministerio de Salud con su Biblioteca y con la Unidad de Patrimonio; Fondo Nacional de Salud (Fonasa); Biblioteca Central de Medicina, Escuela de Salud Pública y Museo Nacional de Medicina de la Universidad de Chile; Sociedad Chilena de Historia de la Medicina; CONICYT; Cuadernos Médico Sociales/ Colegio Médico de Chile y Red ARPA de Archivos Documentales de Valparaíso. El objetivo es contribuir a registrar, organizar y almacenar en formato electrónico las bases del conocimiento e información patrimonial en salud, para hacerla accesible, en forma universal, en un espacio virtual de Internet formado por una red de fuentes de información especializada. Ello se expresará en la Red Chilena de Historia y Patrimonio Cultural de la Salud en el contexto latinoamericano ${ }^{8,9}$.

Otro de los desafíos que nos enfrentamos con miras al Bicentenario es la creación y desarrollo del Centro Nacional de Recuperación y Difusión del Patrimonio Cultural de la Salud, actualmente en etapa inicial de adecuación estructural y funcional en el Antiguo Hospital San José de Santiago, donde está ubicada la sede central de la Unidad de Patrimonio Cultural de la Salud. Tendrá espacios adecuados para exponer muestras de documentos históricos, fotografías y archivos documentales, además de un auditorio patrimonial en salud. Todo ello contribuirá a valorizar el rescate de la memoria histórica y del patrimonio cultural, tanto material como inmaterial, para participar activamente en la formulación y desarrollo de las políticas nacionales, regionales y locales de salud pública en el siglo XXI.

\section{COMENTARIO FINAL}

La historia de la Salud Pública chilena tiene un valor trascendente para las futuras generaciones, en la medida que logremos recuperar y rescatar adecuadamente el riquísimo patrimonio cultural e intercultural que se ha ido construyendo a través de los años y por sucesivas 
generaciones.

Federico Mayor, ex Director General de UNESCO, ha expresado que "el dilema que todas las naciones del planeta afrontan actualmente es el de la necesidad de preservar el pasado sin dejar de construir el futuro, de conciliar el desarrollo con la cultura". Asimismo, ha señalado que "salvaguardar el pasado es importante sólo en la medida en que ese pasado contribuya a renovar el futuro"10.

La importancia de rescatar el patrimonio cultural reside en el hecho que está formado por los bienes culturales que la historia le ha legado a una nación, y por aquellos que en el presente se crean y a los que la sociedad les otorga una especial importancia histórica, científica, simbólica o estética. Es, por consiguiente, la herencia recibida de nuestros antepasados, y que viene a ser el testimonio de su existencia, de su visión de mundo, de sus formas de vida $\mathrm{y}$ de su manera de ser.

Reconocemos que hay distintas facetas del patrimonio cultural de la salud, tangible e intangible, material e inmaterial, que se han ido consolidando en la primera Red Nacional de Responsables del Patrimonio Cultural en Salud y en diferentes instancias descentralizadas del Sistema Nacional de Servicios de Salud.

Con la experiencia acumulada se está en condiciones para los próximos años de profundizar una Política Pública Patrimonial en Salud que contribuya al rescate y valorización del patrimonio histórico y cultural, con acceso libre y gratuito a un verdadero museo de la salud interactivo y descentralizado; un ordenamiento de los archivos y documentos patrimoniales; una recuperación progresiva de los aportes históricos en salud de las generaciones pasadas y presentes; un reencuentro efectivo con el patrimonio cultural intangible; una comunidad que aprecie efectivamente el valor real del patrimonio cultural en el Sistema Nacional de
Servicios de Salud y un verdadero enfoque intercultural en lo patrimonial.

Del conjunto del Estado y la sociedad civil dependerá construir solidaria y colectivamente el patrimonio cultural como "la memoria del porvenir". Es una tarea difícil, pero atractiva; así como larga, pero posible de ser visualizada en el corto plazo.

\section{REFERENCIAS}

1. MINISTERIO DE SALUD. "MEMORIA 2000- 2006. El presente y futuro del patrimonio cultural del Ministerio de Salud".Unidad de Patrimonio Cultural de la Salud- Santiago, Chile, marzo de 2006. (63 páginas)

2. MINISTERIO DE SALUD. Testimonios del Primer Encuentro de Responsables del Patrimonio Cultural del Sistema Nacional de Servicios de Salud. Transcripción de ponencias, comentarios, experiencias y aportes de los participantes. Antiguo Hospital San José. Santiago, Chile, 26-28 de agosto de 2004. Unidad de Patrimonio Cultural de la Salud. Santiago, noviembre de 2005 (144 páginas).

3. HEVIA PATRICIO. Patrimonio Cultural de la Salud. An chil hist med 2006:16: 97-101

4. Portal de la Biblioteca Virtual en Salud de Chile (www.bvs.cl)

5. Portal de la Biblioteca Virtual en Salud de Historia y Patrimonio Cultural de la Salud (http://hpcs.bvsalud.org

6. Grupo de trabajo en BVS4/CRICS7 . Salvador, Bahia, Brasil, septiembre de 2005 http://bvs4.icm19.org/?lang=es.

7. HEVIA, PATRICIO. Historia y patrimonio cultural de salud: propuesta para la creación de un biblioteca virtual latinoamericana. An. Chil. Hist. Med. 2006; 16: 237 - 242

8. HEVIA, PATRICIO. El inicio de la Biblioteca Virtual en Salud de Historia y Patrimonio Cultural de la Salud: Constitución de la Red Latinoamericana desde Santiago de Chile. An chil hist med 2007; 17. 241-249.

9. PACKER, ABEL. BIREME y la BVS frente al futuro".Newsletter BVS 086 21/enero/2009 http://newsletter.bireme.br/new/?lang=es

10. MAYOR, FEDERICO. El Patrimonio, memoria del porvenir. El Correo de la UNESCO. Septiembre 1997 (Páginas 42-43).

Recepción: 27 de noviembre de 2008 Aprobación: 8 de enero de 2009

Usted puede comentar éste y otros artículos publicados en la Revista Chilena de Salud Pública, enviando un correo electrónico a revistasp@med.uchile.cl 